\title{
Human Ad Hoc Networks using Telepathic Connections
}

\section{Yupapin PP1,2*}

${ }^{1}$ Advanced Studies Center, Department of Physics, Faculty of Science, King Mongkut's Institute of Technology Ladkrabang (KMITL), Bangkok 10520, Thailand ${ }^{2}$ Quantum Life Institute, Supakorn Biz-town, Sainoi, Nonthaburi 11150, Thailand

Generally, human communications can be linked by way of feelings, emotions, thoughts, images and desires, where some people can communicate telepathically when dreaming. Clairvoyance is the term given to a message received in a visual form. While hearing an inner voice is referred to as clairaudience. A feeling is termed as clairsentience, and par cognition refers to an instant knowing of something. Telepathy describes receiving thoughts or feelings from another person over distance, without using one of the five typical senses of sight, sound, touch, taste or smell, which is more likely to happen between people who have an existing close relationship. In this concept paper, the ad hoc network is used incorporating the human telepathic connections, which has been the interesting system for short range communication link $[1,2]$, where the advantage is that the communication among small group of people with security and privacy is plausible, in which the dynamic network, for instance, sport rally network, vehicular network, conference participant network, community network and modern social network can also be implemented. Systematically, the ad hoc network is required the mobile communication/electronic instruments including the central controller (dynamic server). Human ad hoc network can be formed by such a system that can provide the short range dynamic network among people, where the basic communication requirement is a brain signal, where the required commands are generated and linked to the information, which is propagated via the whispering gallery mode of coherent light (brain signal) to the required destination [3]. This is allowed the closed loop of communication, which is required the electronic instruments such as amplifier, filter, blue tooth and transceiver with nano-antenna (thin film) [4].

Basically, brain signal is used as telepathic communication medium, which is formed by coherent light in holographic locations. The required brain signals can project to cells (nerves) when the required functions are assigned [5-7], the signal concentration can be formed by the whispering gallery mode, which can be amplified for higher field strength before propagation via a thin film antenna to the destination, where the signal modulation and filter are required. Recently, telepathic communication has shown the promising results [8-10], where the individual telepathic signals can form the short range communication link, which is shown by the model in Figure 1 [11]. The ability to send to and receive from each other thoughts and feelings comes from a spiritual connection between the two of people. The original telepathic signal strength can be increased by using the electronic instruments such as amplifier, which is modulated and

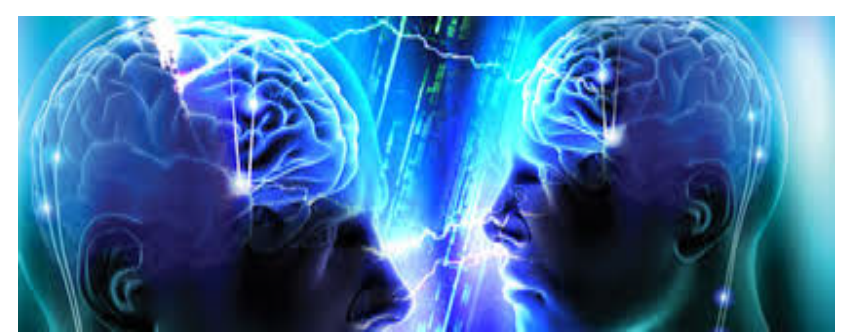

Figure 1: Short range telepathic communication link. transmitted via a nano-antenna, where the up and down conversion link can be operated and formed the communication.

Brain signal can generate the specific Rabi oscillation frequency [12-14], which will be harder for others to sense. People psychically put up a shield to prevent them from seeing his/her intentions. This is namely as code (quantum code, qubit) that can be used for protection, i.e. security. Generally, someone of a stronger mind and greater clarity of thinking can penetrate to the psychic shields of others and see what they are thinking. When two people are spiritually close, they trust each other and they have mutual empathy, where the code is given for communication. Empathy is one of the key ingredients of telepathy. Telepathy which is of the psychic level of the mind tends to operate beyond pure words and linguistics, which takes place in the form of feelings, images and desires. People may be able to receive telepathic communication from another person in the form of pure words. But usually it comes through a feeling, image or desire.

In principle, telepathic communication model consists of two directions, sending and receiving, where two people are able to communicate without being in contact physically, speak to each other through telepathy. The ability to send to and receive from each other thoughts and feelings comes from a spiritual connection between them. When two people are spiritually close to each other, they are often able to sense what the other is feeling or thinking. In operation, the Rabi oscillation frequency can be formed the up and down links by the transceivers between users, while the ad hoc network can be formed by using telepathic telephony network. Brain signals are modulated and transmitted by the nano-antenna arrays to the destinations, the required signals can be accepted and demodulated by the end user antennas, where finally, the corrected signals can be connected by cells and nerves. In Figure 2 [11], the transceiver with amplifier is formed by a blue tooth tag, which is linked between brain signals and thin film nano-antenna.

Human ad hoc network can be implemented by using the short range wireless communication network, where the group of users can be linked and communicated dynamically, where the controlled unit (server) is existed and moved among the users. In Figure 3a [11], the individual telepathic signals can be monitored and linked by the brain and computer interfacing instruments, where the telepathic network can be formed as shown in Figure 3b [11], which is controlled and linked by the central server. The important issue of telepathic

*Corresponding author: Yupapin PP, Advanced Studies Center, Department of Physics, Faculty of Science, King Mongkut's Institute of Technology Ladkrabang, Bangkok (KMITL), Thailand, Tel: +66 02329 80; E-mail: kypreech@kmitl.ac.th

Received March 06, 2014; Accepted March 07, 2014; Published March 13, 2014

Citation: Yupapin PP (2014) Human Ad Hoc Networks using Telepathic Connections. J Biosens Bioelectron 5: e129. doi:10.4172/2155-6210.1000e129

Copyright: ( 2014 Yupapin PP. This is an open-access article distributed under the terms of the Creative Commons Attribution License, which permits unrestricted use, distribution, and reproduction in any medium, provided the original author and source are credited. 


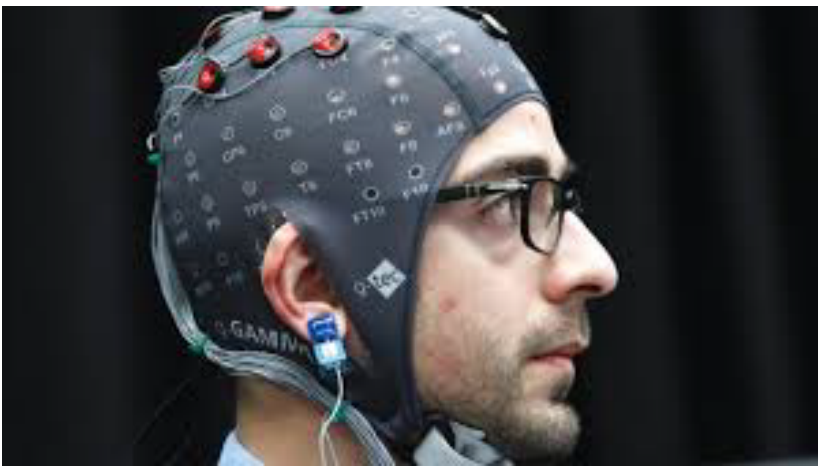

Figure 2: Telepathic telephony model, where thin film optical transceiver (fore head tag) can be used incorporating blue tooth (ear tag) for each user.

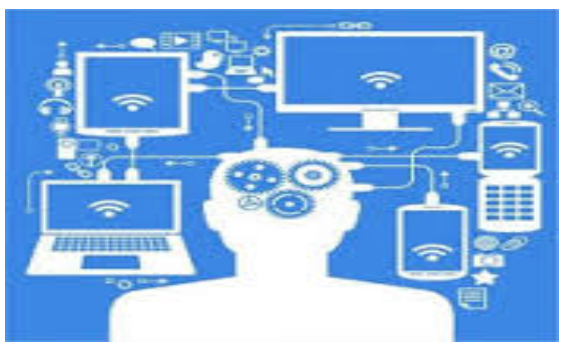

(a)

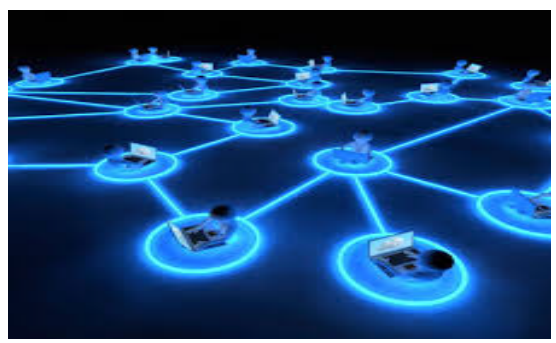

(b)

Figure 3: Human ad hoc networks, where (a) telepathic server(b) ad hoc networks.

communication network is the awareness, where lack of awareness, skepticism and general societal views are often big factors in people locking out this special intuitive ability. It is possible to block others from being able to send and receive telepathic communications, in which the codes (qubits) can be automatically established by each user.

In practice, the telepathic signal strength can be improved by people meditation, which can help to relax the mind and body and make people more receptive to receiving and sending telepathic communications. The noisy signals are almost neglected during the perfect meditation, thus, the telepathic signal becomes the stronger signals in this situation. It also assists with ridding people mind of unwanted and unnecessary thoughts. The open mindedness enhances the chances of telepathic communications, while a closed mind is less likely to be able to transmit or receive effectively. The original telepathic signal without noise is able to link for long distance, for instance, Buddhist saint can have such ability.
The human contact memory is established by the first registration during the first perception and contact between two people (Alice: Sender and Bob: End user), which will be kept (allocated) within the human brain space permanently. The security codes between them will be generated and the information blocked by their security codes if the end user (Bob) is not the required one. On the other hand, the unlocked information will be transmitted if the required end user is confirmed by the feedback brain signals (spirit signals), i.e. telepathic link, where finally, the required security codes will be opened and the information will be transmitted. Moreover, the quantum flip-flop signals can also be generated by using a coherent light source propagates within micro-optical device, where the Rabi oscillation frequency can be established for the started/stopped transmission bits security, in which the stream of information can be securely transmitted in the telepathic communication.

The scanning image information will be recognized of the required end users by million brain cells, where they will be addressed within the brain memory by many layers and sub-layers in the brain cloud computing system [14]. The image recovery can be done by the image pattern recognition recovery, in which the probability of image pattern recognition, where the identical image probability is the criteria of recovery decision. The projection of coherent signals will bring the de-coherent states, where the collapse of waves will bring the required results (required image recovery).

In conclusion, we have proposed that human ad hoc network can be performed by telepathic telephone connections, which can be used as dynamic voiceless networks by using the thin film electronic instruments such as transceiver, nano-antenna and amplifier, where the amplified and modulated signal parts can be constructed by the voiceless mobile telephone system, which can be useful for deaf people. However, the privacy is also needed to clarify and protect, which will be the important issue of required technology. In general, this kind of network will be constructed and popularly used in the near future.

\section{Acknowledgment}

The authors would like to acknowledge the King Mongkut's Institute of Technology Ladkrabang (KMITL), Bangkok 10520, Thailand for giving the research facilities.

\section{References}

1. Bunruangses M, Sunat K, Mitatha S, Yupapin PP (2010) Vehicular ad hoc network for a surveillance system using multi-frequency band enhancement. Opt. Eng. 49, 095001-1-7.

2. Frodigh $M$, Johansson $P$, Larsson $P$ (2000) Wireless ad hoc networks-The art of networking without a network. Ericson review 4:248-263.

3. Yupapin PP (2013) Nonlinear coupling effects of waves in a panda ring. Science Discovery 1: 1-5.

4. Thammawongsa N, Moonfangklang N, Mitatha S, YupapinPP (2012) Novel nano-antenna system design using photonic spin in a panda ring resonator. PIER Lett 31: 75-87.

5. Yupapin PP, Thammawongsa N, Ali J (2014) Consciousness and subconsciousness detection model under Cerenkov radiation. Microw. And Opt. Techn.Lett. In press.

6. Tamee K, Chaiwong K, Yothapakdee K, Yupapin PP (2013) Brain signal monitoring and encoding for humanoid robots use. $\mathrm{J}$ Biosensors and Bioelectronics 4: e124. 
Citation: Yupapin PP (2014) Human Ad Hoc Networks using Telepathic Connections. J Biosens Bioelectron 5: e129. doi:10.4172/21556210.1000 e129

Page 3 of 3

7. Ali J, Yupapin PP (2014) Micro-Cloud Computing System by Human Quantum Computer. J Biosensors and Bioelectronics 5: e127.

8. Das P, Purkayastha BS (2010) Development telepathic power of network using neural network approach. Wireless Communication and Sensor Computing, ICWCSC 2010. International Conference on: 1-5.

9. Sheldrake R, Avraamides L (2009) An automated test for telepathy in connection with emails. J Scientific Exploration 23: 29-36.

10. Campbell AT, Choudhury T, Hu S, Lu H, Mukerjee MK (2010) NeuroPhone: Brain mobile phone interface using a wireless EEG headset. MobiHeld 2010, New Delhi, India.
11. Telepathic communication, Google search, March, 2014.

12. Yupapin PP, Pantian S, Ali J (2014) Novel design Rabi oscillation system for human quantum life detection probe. Life Sci J 11: 235-243.

13. Pantian S, Yupapin PP (2013) THz Rabi frequency oscillation for human consciousness/sub-consciousness detection probe use. J Biosensors and Bioelectronics 4: e126.

14. Ali J and Yupapin PP (2014) Micro-cloud computing system by human quantum computer, J Biosensors and Bioelectronics, 5(1), e127. 